ИЗВЕСТИЯ АКАДЕМИИ НАУК ЭСТОНСКОИ ССР. ФИЗИКА * МАТЕМАТИКА

PROCEEDINGS OF THE ACADEMY OF SCIENCES OF THE ESTONIAN SSR. PHYSICS * MATHEMATICS

$1985,34,2$

\title{
К ИССЛЕДОВАНИЮ НАПРЯЖЕНИЙ В ТРЕХМЕРНЫХ КУБИЧЕСКИХ КРИСТАЛЛАХ МЕТОДОМ ФОТОУПРУГОСТИ
}

\author{
(Представил Х. Абен)
}

\section{1. Введение}

Основные уравнения теории пьезооптического эффекта для оптически анизотропных тел приведены в $\left[{ }^{1-4}\right]$. В $\left[{ }^{5}\right]$ тензор диэлектрической непроницаемости рассматривается как линейная функция двух аргументов: тензора деформации и тензора напряжений. Предложенная в этой работе методика позволяет изучать некоторые особенности метода фотоупругости различных кристаллографических классов кубической сингонии.

Изучению плоской задачи для пластинок, параллельных плоскостям (100) и $(110)$, посвящены $\left[{ }^{6-8}\right]$, а исследованию температурных напряжений $-\left[{ }^{9,10}\right]$. В [ $\left.{ }^{11}\right]$ исследуется распределение температурных напряжений в пластинке германия. Предполагается, что напряженное состояние осесимметричное и по экспериментально найденной эпюре напряжений восстанавливается температурное поле, имеющее место при выращивании кристалла.

В $\left[{ }^{12}\right]$ предлагается методика разделения главных напряжений в произвольно ориентированной относительно кристаллографических осей пластинке. Эта же методика рассматривается в $\left.{ }^{13}\right]$, но в отличие от $\left[{ }^{12}\right]$ в последней коэффициенты уравнений пьезооптического эффекта приведены в зависимости от кристаллографических индексов.

Обобщенные выражения для описания индуцированного двойного лучепреломления получены в $\left[{ }^{14}\right]$. В предположении, что в кристалле на пути света отсутствует вращение квазиглавных направлений диэлектрического тензора, определяются усредненные напряжения по толщине кристалла.

В настоящей работе выводятся в матричном виде оптические уравнения метода фотоупругости кубических кристаллов для случая просвечивания кристалла в произвольном направлении. Рассматривается определение характеристических величин $\left[{ }^{15}\right]$ при прохождении света через кубический кристалл.

\section{2. Вывод основных формул}

Используем следующие декартовы системы координат. Матрицу пьезооптических коэффициентов $\pi\left[{ }^{2}\right]$ опишем в системе координат $X$, совпадающей с кристаллографическими направлениями [100], [010] и [001]. Напряжения выразим в системе координат $X^{\prime}$, которая связана с внеш- 
ней геометрией кристалла. Координатную систему $X^{\prime \prime}$ выберем так, чтобы одна из ее осей совпадала с направлением просвечивания. Компоненты векторов и тензоров обозначим апострофами, которые соответствуют использованной системе координат.

Преобразование систем координат производим с помощью матриц направляющих косинусов

$$
\begin{aligned}
& x_{i}^{\prime}=a_{i j} x_{j}, \\
& x_{i}^{\prime \prime}=b_{i j} x_{j^{*}}^{\prime}
\end{aligned}
$$

В этом случае компоненты тензоров пьезооптических коэффициентов и напряжений, записанные в сокращенной форме, преобразуются $\left[{ }^{16}\right]$ по правилам

$$
\begin{aligned}
& \Pi_{m n}^{\prime}=A_{m l} \Pi_{l p} A_{p n}^{-1},- \\
& \sigma_{m}^{\prime}=A_{m l} \sigma_{l},
\end{aligned}
$$

или в матричной форме

$$
\begin{aligned}
& \Pi^{\prime}=A \Pi A^{-1}, \\
& \sigma^{\prime}=A \sigma, \\
& \Pi^{\prime \prime}=B \Pi^{\prime} B^{-1}, \\
& \sigma^{\prime \prime}=B \sigma^{\prime} .
\end{aligned}
$$

Здесь П, П', П" $A, B-6 \times 6$-матрицы, $\sigma, \sigma^{\prime}, \sigma^{\prime \prime}-6 \times 1$-матрицы. Матрицы $A$ и $B$ различаются только тем, что элементами матрицы $A$ являются элементы матрицы направляющих косинусов $a_{i j}$, а матрицы $B-b_{i j}$.

Выделим из кристалла, в котором имеет место трехмерное напряженное состояние, тонкий слой толщиной $\mathrm{d} x_{i}{ }^{\prime \prime}$. Предположим, что по толщине слоя напряжения постоянны, и отсутствует вращение квазиглавных направлений диэлектрического тензора.

Изменение диэлектрической непроницаемости $\Delta \xi^{\prime \prime}$ под влиянием напряжений $\sigma^{\prime \prime}$ определяется соотношением $\left[{ }^{2}\right]$

$$
\Delta \xi^{\prime \prime}=\Pi^{\prime \prime} \sigma^{\prime \prime} .
$$

При просвечивании тонкого слоя вдоль оси $x_{i}^{\prime \prime}$, которая перпендикулярна рассматриваемому слою, разность хода $\mathrm{d} \delta_{i}{ }^{\prime \prime}$ и азимут квазиглавного направления диэлектрического тензора (оптическая изоклина) $\alpha_{i}^{\prime \prime}$ связаны с элементами матрицы $\Delta \xi^{\prime \prime}$ следующими выражениями $\left[{ }^{3,4}\right]$

$$
\begin{aligned}
& \mathrm{d} \delta_{i} \cos 2 \alpha_{i}^{\prime \prime}=\left(n_{0}^{3} / 2\right)\left(\Delta \xi_{i+1}^{\prime \prime}-\Delta \xi_{i+2}^{\prime \prime}\right) \mathrm{d} x_{i}^{\prime \prime}, \quad(i+3 \equiv i) \\
& \mathrm{d} \delta_{i} \sin 2 \alpha_{i}^{\prime \prime}=n_{0}^{3} \Delta \xi_{i+3}^{\prime \prime} \mathrm{d} x_{i}^{\prime \prime}, \quad(i=1,2,3),
\end{aligned}
$$

где $n_{0}$ - показатель преломления ненапряженного материала.

Выражения (7) можно записать в матричном виде

$$
\Psi_{i}^{\prime \prime}=\left(n_{0}^{3} / 2\right) Q_{i} \Delta \xi^{\prime \prime},
$$

где матрицы $\Psi_{i}^{\prime \prime}, Q_{i}$ и $\Delta \xi^{\prime \prime}$ имеют следующий вид:

$$
\Psi_{i}^{\prime \prime}=\left(\begin{array}{l}
\mathrm{d} \delta_{i}^{\prime \prime} \cos 2 \alpha_{i}^{\prime \prime} / \mathrm{d} x_{i}^{\prime \prime} \\
\mathrm{d} \delta_{i}^{\prime \prime} \sin 2 \alpha_{i}^{\prime \prime} / \mathrm{d} x_{i}^{\prime \prime}
\end{array}\right) .
$$




$$
\begin{gathered}
Q_{1}=\left(\begin{array}{rrrrrr}
0 & 1 & -1 & 0 & 0 & 0 \\
0 & 0 & 0 & 2 & 0 & 0
\end{array}\right), \\
Q_{2}=\left(\begin{array}{rrrrrr}
-1 & 0 & 1 & 0 & 0 & 0 \\
0 & 0 & 0 & 0 & 2 & 0
\end{array}\right) \\
Q_{3}=\left(\begin{array}{rrrrrr}
1 & -1 & 0 & 0 & 0 & 0 \\
0 & 0 & 0 & 0 & 0 & 2
\end{array}\right) \\
\Delta \xi^{\prime \prime}=\left(\begin{array}{c}
\Delta \xi_{1}^{\prime \prime} \\
\Delta \xi_{2}^{\prime \prime} \\
\vdots \\
\Delta \xi_{6}^{\prime \prime}
\end{array}\right)
\end{gathered}
$$

Подставляя соотношения (4), (5) и (6) в формулу (8), получим

$$
\Psi_{i}^{\prime \prime}=\left(n_{0}^{3} / 2\right) Q_{i} B A \Pi A^{-1} \sigma^{\prime} .
$$

Для кубических кристаллов порядок матриц в выражении можно снизить от шести до пяти

$$
\Psi_{i}^{\prime \prime}=Q_{i}^{*} B^{*} A^{*} C^{*} A^{-1^{*}} \sigma^{\prime *} .
$$

Здесь элементы матриц $Q_{i}{ }^{*}$ и $\sigma^{\prime *}$ вычисляются по формулам

$$
\begin{array}{ll}
q_{j, k}^{*}=q_{j, k+1}, & (j=1,2 ; k=1 ; 2, \ldots, 5) \\
\sigma_{j}^{\prime *}=\sigma_{j+1}^{\prime}-\sigma_{1}^{\prime}, & (j=1,2) \\
\sigma_{j}^{\prime *}=\sigma_{j+1}^{\prime}, & (j=3,4,5)
\end{array}
$$

и элементы матриц $A^{*}, A^{-1 *}$ и $B^{*}$ имеют общее свойство

$$
a_{j, k}^{*}= \begin{cases}a_{j+1, k+1}-a_{1, k+1}, & (j=1,2 ; k=1,2, \ldots, 5) \\ a_{j+1, k+1}, & (j=3,4,5) .\end{cases}
$$

Матрица $C^{*}$ для кубических кристаллов имеет вид

$$
C^{*}=\left(n_{0}^{3} / 2\right)\left(\begin{array}{ccccc}
\pi_{11}-\pi_{12} & \pi_{12}-\pi_{13} & 0 & 0 & 0 \\
\pi_{13}-\pi_{12} & \pi_{11}-\pi_{13} & 0 & 0 & 0 \\
0 & 0 & \pi_{44} & 0 & 0 \\
0 & 0 & 0 & \pi_{44} & 0 \\
0 & 0 & 0 & 0 & \pi_{44}
\end{array}\right) .
$$

Для случая, когда свет распространяется вдоль оси $x_{1}^{\prime \prime}$, уравнение (13) можно переписать в виде

$$
\begin{aligned}
& \mathrm{d} \delta_{1}^{\prime \prime} \cos 2 \alpha_{1}^{\prime \prime}=\left[\sum_{m=1}^{2} E_{m}\left(\sigma_{m+1}^{\prime}-\sigma_{1}^{\prime}\right)+\sum_{m=3}^{5} E_{m} \sigma_{m+1}^{\prime}\right] \mathrm{d} x_{1}^{\prime \prime}, \\
& \mathrm{d} \delta_{1}^{\prime \prime} \sin 2 \alpha_{1}^{\prime \prime}=\left[\sum_{m=1}^{2} F_{m}\left(\sigma_{m+1}^{\prime}-\sigma_{1}^{\prime}\right)+\sum_{m=3}^{5} F_{m} \sigma_{m+1}^{\prime}\right] \mathrm{d} x_{1}^{\prime \prime} ;
\end{aligned}
$$

где коэффициенты $E_{m}, F_{m}$ вычисляются из матричного произведения

$$
\begin{aligned}
& E_{m}=\left(Q_{1}^{*} B^{*} A^{*} C^{*} A^{-1^{*}}\right)_{1, m}, \\
& F_{m}=\left(Q_{1}^{*} B^{*} A^{*} C^{*} A^{-1 *}\right)_{2, m} .
\end{aligned}
$$




\section{3. Определение характеристических величин}

В общем случае при просвечивании произвольно ориентированного относительно кристаллографических осей кубического кристалла квазиглавное направление диэлектрического тензора на пути света вращается. B $\left[{ }^{17}\right]$ показывается, что при слабом вращении квазиглавных направлений можно интегрировать уравнения (17). На основе предположения слабого вращения квазиглавных направлений в $\left[{ }^{17,18}\right]$ разработана методика определения остаточных напряжений в кубических кристаллах призматического и кругового поперечного сечения.

В случае, если вращение квазиглавных направлений диэлектрического тензора на световом луче значительное, фотоупругая модель для одного луча света полностью характеризуется тремя величинами: первичными и вторичными характеристическими направлениями и характеристической разностью фаз. Для их определения фотоупругая модель мысленно разделяется на $k$ двупреломляющие пластинки толщиной $t_{j}$, которые характеризуются матрицей Джонса $\left[{ }^{19}\right]$

$$
U_{j}=\cos \left(\Delta_{j} / 2\right)\left(\begin{array}{ll}
1 & 0 \\
0 & 1
\end{array}\right)+i \sin \left(\Delta_{j} / 2\right)\left(\begin{array}{rr}
\cos 2 \varphi_{j} & \sin 2 \varphi_{j} \\
\sin 2 \varphi_{j} & -\cos 2 \varphi_{j}
\end{array}\right) .
$$

При исследовании кубических кристаллов разность фаз $\Delta_{j}$ и квазиглавное направление $\varphi_{j}$ определяются из уравнений (17)

$$
\begin{aligned}
& \Delta_{j}=\left(360^{\circ} / \lambda\right) \sqrt{M_{j}^{2}+N_{j}^{2}}, \\
& \varphi_{j}=0,5 \operatorname{arctg}\left(N_{j} / M_{j}\right),
\end{aligned}
$$

где

$$
\begin{aligned}
& M_{j}=\left[\sum_{m=1}^{2} E_{m}\left(\sigma_{m+1}^{\prime}-\sigma_{1}^{\prime}\right)+\sum_{m=3}^{5} E_{m} \sigma_{m+1}^{\prime}\right] t_{j}, \\
& N_{j}=\left[\sum_{m=1}^{2} F_{m}\left(\sigma_{m+1}^{\prime}-\sigma_{1}^{\prime}\right)+\sum_{m=3}^{5} F_{m} \sigma_{m+1}^{\prime}\right] t_{j},
\end{aligned}
$$

$\lambda$ - длина волны света.

Матрицу фотоупругой модели получаем умножением матриц пластинок (19)

$$
U=U_{k} U_{k-1} \ldots U_{2} U_{1} .
$$

Из матрицы $U$, которая имеет вид

$$
U=\left(\begin{array}{cc}
p+i q & r+i s \\
-r+i s & p-i q
\end{array}\right),
$$

можно вычислить первичные и вторичные характеристические углы $\alpha_{0}$, $\alpha_{*}$, а также характеристическую разность фаз $\Delta_{*}$ по формулам [ $\left.{ }^{15}\right]$

$$
\begin{aligned}
& \operatorname{tg} 2 \alpha_{0}=(p s+q r) /(p q-r s), \\
& \operatorname{tg} 2 \alpha_{\star}=(p s-q r) /(p q+r s), \\
& \cos \Delta_{\star}=p^{2}+r^{2}-q^{2}-s^{2} .
\end{aligned}
$$

\section{4. Численный пример}

В качестве примера рассмотрим цилиндр из кубического монокристалла, торцы которого параллельны плоскостям (111) (рисунок). Пусть в ци- 
линдре имеет место осесимметричное напряженное состояние без градиента напряжений вдоль оси цилиндра. Напряжения аппроксимируем полиномами

$$
\begin{aligned}
& \sigma_{z}=c_{0}+c_{2} \varrho^{2}+c_{4} \mathrm{Q}^{4}, \\
& \sigma_{r}=c_{0} / 2+c_{2} \varrho^{2} / 4+c_{4} \mathrm{Q}^{4} / 6, \\
& \sigma_{\theta}=c_{0} / 2+3 c_{2} \mathrm{Q}^{2} / 4+5 c_{4} \mathrm{Q}^{4} / 6
\end{aligned}
$$

Приведем результаты вычиолений характеристических величин для случая, когда угол между направлениями просвечивания $x_{1}{ }^{\prime \prime}$ и осью $x_{1}{ }^{\prime \prime} \theta=45^{\circ}$. Поперечное сечение цилиндра разделялось на двадцать пластинок. Величины разности фаз $\Delta_{j}$ и квазиглавных направлений $\varphi_{j}$ для каждой пластинки при $x_{2}^{\prime \prime}=0$ и $x_{2}=10$ мм представлены в таблице.

Из этих результатов видно, что при просвечивании вдоль оси $x_{1}{ }^{\prime \prime}\left(x_{2}^{\prime \prime}=0\right)$, распределение разности фаз и квазиглавного направления симметричное. Несмотря на то, что внутри модели имеется значительное вращение ква-

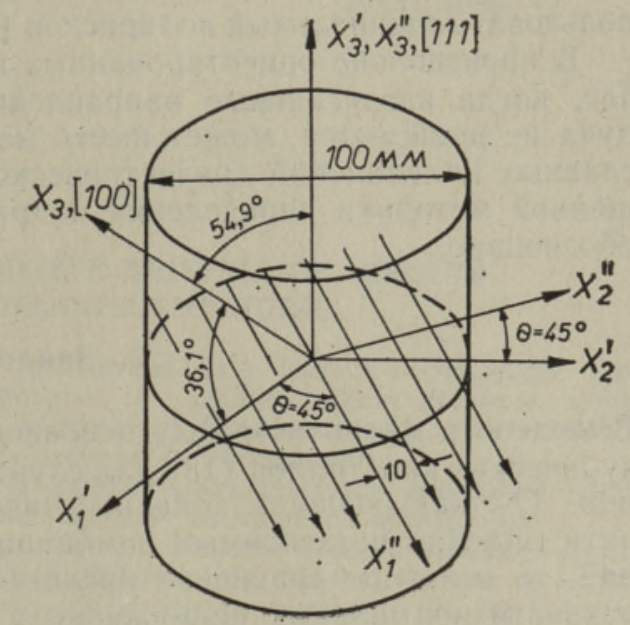

Схема просвечивания цилиндра.

\begin{tabular}{|c|c|c|c|c|}
\hline \multirow{2}{*}{$j$} & \multicolumn{2}{|c|}{$x_{2}^{\prime \prime}=0 \mathrm{MM}$} & \multicolumn{2}{|c|}{$x_{2}{ }^{\prime \prime}=10$ мм } \\
\hline & $\Delta_{j}$, град & $\varphi_{j}$, град & $\Delta_{j}$, град & $\varphi_{j}$, град \\
\hline $\begin{array}{r}1 \\
2 \\
3 \\
4 \\
5 \\
6 \\
7 \\
8 \\
9 \\
10 \\
11 \\
12 \\
13 \\
14 \\
15 \\
16 \\
17 \\
18 \\
19 \\
20 \\
\end{array}$ & $\begin{array}{l}5,259 \\
3,982 \\
3,329 \\
3,379 \\
3,877 \\
4,521 \\
5,131 \\
5,627 \\
5,970 \\
6,147 \\
6,147 \\
5,970 \\
5,627 \\
5,131 \\
4,521 \\
3,877 \\
3,379 \\
3,329 \\
3,982 \\
5,259\end{array}$ & $\begin{array}{l}125,203 \\
133,627 \\
145,670 \\
158,149 \\
167,219 \\
172,820 \\
176,199 \\
178,235 \\
179,402 \\
179,936 \\
179,936 \\
179,402 \\
178,235 \\
176,199 \\
172,820 \\
167,219 \\
158,149 \\
145,670 \\
133,627 \\
125,203\end{array}$ & $\begin{array}{l}3,558 \\
2,047 \\
1,364 \\
1,946 \\
2,981 \\
3,764 \\
4,461 \\
5,019 \\
5,069 \\
5,536 \\
5,536 \\
5,050 \\
5,044 \\
4,635 \\
4,810 \\
3,920 \\
3,989 \\
4,571 \\
5,680 \\
7,219\end{array}$ & $\begin{array}{r}114,061 \\
123,462 \\
146,004 \\
169,844 \\
177,670 \\
0,738 \\
2,039 \\
2,457 \\
2,296 \\
1,652 \\
0,504 \\
178,740 \\
176,132 \\
172,291 \\
166,633 \\
158,610 \\
148,628 \\
138,761 \\
130,940 \\
125,402\end{array}$ \\
\hline & $\alpha_{1}=\alpha_{*}$ & $\begin{array}{r}93,719^{\circ} \\
93,719^{\circ} \\
162,801^{\circ} \\
0\end{array}$ & $\alpha=\alpha_{*}$ & $\begin{array}{r}138,354^{\circ} \\
38,047^{\circ} \\
117,971^{\circ} \\
-100,307^{\circ}\end{array}$ \\
\hline
\end{tabular}

зиглавных направлений, характеристические углы $\alpha_{0}$ и $\alpha_{*}$ совпадают. Характеристический угол 


$$
\alpha=\alpha_{\star}-\alpha_{0}
$$

равняется нулю, и экспериментальные исследования можно провести на обычном скрещенном полярископе. Нужно иметь в виду, что теперь параметр изоклины соответствует первичному характеристическому углу и при измерении разности фаз получим характеристическую разность фаз.

Во втором случае, когда $x_{2}^{\prime \prime}=10$ мм характеристический угол не равняется нулю и для измерения характеристических величин нужно использовать специальный полярископ $\left[{ }^{15}\right]$.

В произвольно ориентированных кубических кристаллах даже в случае, когда квазиглавные направления напряжений на пути светового луча не вращаются, может иметь место существенное вращение квазиглавных направлений диэлектрического тензора, и использование упрощенной методики определения напряжений $\left[{ }^{17,}{ }^{18}\right]$ в данном случае не обосновано.

\section{5. Заключение}

Выведено в матричном виде основное уравнение метода фотоупругости кубических кристаллов (13) для случая объемного напряженного состояния. Так как разность хода и оптическая изоклина зависят только от пяти линейно независимых комбинаций компонентов тензора напряжений, то основное уравнение представлено в виде, содержащем только разности нормальных напряжений и касательные напряжения.

Рассматривается вычисление характеристических величин в случаях, когда при просвечивании кубического кристалла на световых лучах имеется сильное вращение квазиглавных направлений диэлектрического тензора.

В качестве примера определены характеристические величины в цилиндре, торцы которого параллельны плоскостям (111). В цилиндре имеет место осесимметричное напряженное состояние (24). Показано, что хотя при просвечивании цилиндра на лучах света нет вращения квазиглавных напряжений, вращение квазиглавных направлений оптической анизотропии имеет место. В случае, когда луч света проходит ось цилиндра, вращение является симметричным относительно этой оси, в противном случае оно несимметричное.

\section{ЛИТЕРАТ У Р А}

1. Pockels. F. Lehrbuch der Kristalloptik. Leipzig, Teubner, 1906.

2. Най Дж. Физические свойства кристаллов. М., ИЛ, 1960.

3. Бугаков И. И., Грах И. И. Вестн. Ленингр. ун-та, № 19, 102-108 (1968).

4. Бугаков Н. Н., Грах И. Н., Конакова Н. С. Тр. VII Всесоюзн, конфер. по поляр.опт. методу иссл. напряжений, 4. Таллин. 1971, 124-127.

5. Малолеткин Г. Н., Фомин В. Л. Мех. тверд. тела, № 6, 81-84 (1974).

6. Краснов В. М., Степанов А. В. Ж. эксперим. и теор. физ., 25, № 1, 98-105 (1953).

7. Житников Р. А., Степанов А. В. Ж. техн. фнз., 26, № 4, 779-786 (1956).

8. Краснов В. М. В кн.: Поляризационно-оптический метод исследования напряжений. Л., изд-во ЛГУ, 1960, 263-268.

9. Сиротин Ю. Н. Кристаллография, 1, № 6, 208-217 (1956)

10. Ннденбом B. Л., Житомирский $H$. С., Чебанова $Г$. С. Кристаллография, 18, № $1,39-48$ (1973).

11. Никитенко В. И., Ннденбом В. Л. Кристаллография, 6, № 3, 432-438 (1961).

12. Афанасьев Н. Н., Грах Н. И. Тр. VII Всесоюзн. конфер. по поляр.-опт. методу нссл. нарпяжений, 4. Таллин, 1971, 134-139. 
13. Каплан М. С., Красовицкая И. М., Сумин В. И. В кн.: Монокристаллы и техника. Харьков, изд-во ВНИИ монокристаллов, № 12, 89-91 (1975).

14. Афанасьев И. И. Оптика и спектроскопия, 55, № 3, 525-531 (1983).

15. Абен Х. К. Интегральная фотоупругость. Таллин, «Валгус», 1975.

16. Bond, B. L. Bell System Techn. J., 22, № 1 (1943).

17. Бросман Э. Изв. АН ЭССР. Физ. Матем., 26, № 4, 457-461 (1977).

18. Aben, H., Brosman, E. VDI-Berichte, № 313, 45-50 (1978).

19. Шерклифф У. Поляризованный свет. М., «Мир», 1975.

Институт кибернетики Академии наук Эстонской ССР
Поступила в редакцию $1 /$ XII 1984

\section{S. IDNURM, J. JOSEPSON}

\section{KOLMEMOOTMELISTE KUUBILISTE KRISTALLIDE PINGETE UURIMISEST FOTOELASTSUSMEETODIL}

Maatriksi kujul on esitatud fotoelastsuse põhivõrrand (13) kuubiliste kristallide ruumilise pingeolukorra uurimiseks. Et käiguvahe. ja optiline isokliin olenevad ainult viiest lineaarselt sōltumatust pingetensori komponendi kombinatsioonist, siis tuletatud põhivõrrand sisaldab üksnes normaalpingete vahesid ja nihkepingeid.

On vaadeldud karakteristlike nurkade ja karakteristliku faasivahe arvutamist juhul, kui kuubilise kristalli läbivalgustamisel esineb optiliste peasuundade pöördumine.

On toodud arvutusnäide karakteristlike suuruste määramise kohta kuubilisest kristallist silindris, milles on telgsümmeetriline pingeolukord (24). Näitest ilmneb, et kuubilistes kristallides võib esineda isegi juhtumeil, kui pingete peasuunad ei pöördu, küllaltki oluline optiliste peasuundade pöoördumine ja seega pole pingete uurimise lihtsustatud metoodika $\left[{ }^{17,18}\right]$ siin rakendatav.

\section{S. IDNURM, J. JOSEPSON}

\section{INVESTIGATION OF STRESSES IN THREE-DIMENSIONAL CUBIC SINGLE CRYSTALS BY PHOTOELASTICITY}

An optical equation of photoelasticity in a matrix form (13) for arbitrary orientated cubic single crystals is derived. As the isoclinic parameter and the relative phase retardation in a crystal are determined by five linear independent combinations of stress tensor components, the equation contains only two normal stress differences and three shear stress components.

Equation (13) (or its equivalence equations $(17-18)$ ) has been used to calculate the characteristic directions and the characteristic phase retardation (23) in a cubic single crystal.

As an example, determination of the characteristic directions in a crystal of cylindrical form which has an axi-symmetric state of stresses (24), is discussed. It comes out from the calculations that even in case of constant quasi-principal stress axes there exists a great rotation of the secondary optical axes, and it is not allowed to use the simplified methods $\left[{ }^{17}, 18\right]$. 